\title{
Estimation de la composition des carcasses de poulains de boucherie à partir de la composition de l'épaule ou d'un morceau monocostal prélevé au niveau de la $14^{e}$ côte
}

\author{
W. MARTIN-ROSSET, R. BOCCARD *, J. ROBELIN, M. JUSSIAUX ** \\ et Catherine TRILLAUD-GEYL ** \\ avec la collaboration technique de N. NICOLAS * \\ Roland JAILLER et Chantal DEHALLE \\ Station des Productions bovines et chevalines \\ * Station de Recherches sur la Viande \\ I.N.R.A., Centre de Recherches de Clermont-Ferrand \\ Theix, Saint-Genès Champanelle, F 63122 Ceyrat \\ ** Institut technique du Cheval \\ Station expérimentale des Haras, F 19230 Arnac-Pompadour
}

\section{Résumé}

75 poulains et pouliches de diverses races lourdes ont été abattus à l'âge de 12, 18, 24, 30 mois et leur carcasse disséquée.

Trois critères susceptibles d'être liés à la composition anatomique de la carcasse ont été mesurés : le poids des pannes, celui des canons et la compacité de la cuisse. Par ailleurs, nous avons prélevé et disséqué un morceau monocostal situé au niveau de la $14^{\circ}$ côte d'une part et l'épaule d'autre part.

Le poids des tissus de la carcasse a été relié aux différents critères mesurés à l'abattage par la technique de régression progressive. Trois équations d'estimation différentes ont été ainsi établies pour chaque tissu. Il a été tenu compte de l'effet de la race et du sexe dans l'équation d'estimation du poids des dépôts adipeux, mais seulement de la race dans le cas du squelette.

Le poids des dépôts adipeux et des muscles sont estimés respectivement à $4,9 \mathrm{~kg}$ et $5,0 \mathrm{~kg}$ près. La précision de l'estimation du poids du squelette est de 3,0 kg.

Mots clés : cheval, composition anatomique, estimation.

\section{Introduction}

Le développement de la production de viande chevaline à partir d'animaux jeunes implique des études sur la croissance et la possibilité de caractériser la composition de la carcasse des animaux abattus. 
La mesure directe de la composition anatomique par dissection complète de la carcasse et pesée des différents tissus est une méthode coûteuse qui ne peut être appliquée en routine à un grand nombre d'animaux. Il faut donc disposer d'une méthode indirecte.

Il n'existe pas de telle méthode chez le cheval. En revanche, depuis les travaux de Callow, 1962, plusieurs auteurs ont cherché à estimer, chez les bovins, la composition de la carcasse à partir d'un morceau monocostal prélevé au niveau de la $11^{\prime \prime}$ côte et d'autres critères facilement mesurables (Ledger \& Hutchinson, 1962 ; Geay \& Beranger, 1969 ; Robelin \& Geay, 1975. Chez les ovins la composition de la carcasse est estimée à l'aide de différents éléments du corps vide, variables selon le tissu considéré (Prud'hon, Reyne \& Garambois, 1972) ou à partir de celle de l'épaulc (Boccard, Dumont, Lefebvre, 1976).

Une étude a été entreprise pour mettre au point de telles méthodes applicables au poulain de boucherie. On a retenu au départ différents critères d'estimation facilement mesurables tels que le poids de la carcasse ou celui des pannes (dépôts adipeux thoraciques adhérant à la face interne du sternum), ou encore la composition tissulaire d'un morceau costal ou celle de l'épaule. Le but de cette étude était de sélectionner les meilleurs critères d'estimation et d'analyser l'incidence de la race et du sexe des animaux sur les équations d'estimation.

\section{Matériel et méthodes}

\section{A. Animaux}

120 poulains et pouliches nés au printemps 1972, appartenant aux 5 principales races de trait françaises (Ardennaise, Boulonnaise, Bretonne, Comtoise, Percheronne) ont été utilisés dans une expérience destinée à décrire la croissance et les caractéristiques des carcasses du cheval de boucherie (MARTIN-Rosset \& Jussiaux, 1977 ; MarTinRosset et al., 1983).

120 de ces animaux ont été abattus aux âges de 12, 18, 24, 30 mois, à raison de 2 animaux par sexe et par race pour chacun d'eux. 75 seulement ont fait l'objet d'une dissection complète.

\section{B. Méthode de mesure}

Les mesures effectuées immédiatement après l'abattage et la méthode de dissection de la demi-carcasse ont été déjà décrites par ailleurs (MARTIN-RosSEt et al., 1983).

On a prélevé l'épaule par section des différents muscles qui la rattachent au reste de la carcasse, au niveau du bord antérosupérieur du cartilage de prolongement du scapulum et des articulations chondro-costale et du bord antérieur du membre. Certains de ces muscles sectionnés dans le corps charnu sont restés en partie sur l'épaule. C'est le cas du peaucier, du trapèze et du grand dorsal qui ont été ensuite séparés totalement de l'épaule. 
Sur le "pan traité » de la demi-carcasse gauche, la $14^{\prime \prime}$ côte constitue l'extrémité postéricure du train de côte. Elle est obtenue après séparation du milieu du train de côtes du " pan traité ", par section au niveau du 14" espace intercostal (cntre la 14" et la $15^{\circ}$ côte) et par section analogue au niveau du $13^{\prime \prime}$ espace intercostal.

Les différents tissus (os, muscles, dépôts adipeux) de l'épaule et de la $14^{\prime \prime}$ côte ont été séparés au couteau et pesés au gramme près. Leur poids a été corrigé respectivement en fonction du poids total de l'épaule ou de la 14" côte, en admettant que la perte d'eau durant la dissection, était répartic proportionnellement au poids de chacun des tissus. Le poids des différents tissus chauds a été calculé comme dans le cas de la carcasse, à partir du poids de ces tissus froids (MARTIN-Rosset el al., 1983).

Par ailleurs, on a mesuré la compacité de la cuisse, rapport entre son épaisscur mesurée en un point précis défini chez les bovins par Dumont, Le Guelte, Arnoulx (1961), et sa longueur (distance comprise entre l'extrémité du jarret et la pointe inférieure de la symphyse).

\section{Méthodes d'analyse statistique}

Une analyse graphique préliminaire des donnécs a d'abord été réaliséc afin de détecter les éventuelles données aberrantes.

Ensuite les résultats ont été analysés par régression progressive intra-race, en utilisant soit la composition de la 14" côte, soit celle de l'épaule. En effet, il n'est pas concevable de proposer des équations d'estimation faisant intervenir sinuláanément ces deux groupes d'estimateurs. C'est pourquoi nous avons effectué pour chaque tissu 2 analyses séparées pour laisser à l'utilisatcur le choix d'employer l'épaule ou la $14^{\prime \prime}$ côtc.

On a déterminé l'incidence de la race et du sexe des animaux sur ces équations grâce à une analyse cle covariance, en utilisant le modèle mathématique décrit par" SEEBECK (1973) et le programme de calcul sur ordinateur mis au point par cet auteur (Robelin \& Geay, 1975).

\section{Résultats}

\section{A. Estimation des dépôts adipeux de la carcasse (tabl. 1 et 2)}

La dissection de la 14" côte permet d'estimer avec la même précision que la dissection de l'épaule, le poids des dépôts adipcux de la carcasse. Les coefficients de variation résiduelle CVR, exprimés en pourcentage du poids des clépôts adipeux sont respectivement égaux à 11,9 ct 12,2 p. 100.

Les équations d'estimation utilisent les covariables conmunes : poids de carcasse chaude et poids des pannes dont l'apport est très hautement significatif $(P<0,001)$. La précision de l'estimation du poids des tissus adipeux à partir de ces deux variables seules est alors de 11 à $24 \mathrm{~kg}$ selon le type génétique. L’introduction d'autres covariables spécifiques telles que le poids de dépôts adipeux et des muscles de la $14^{*}$ côte, ou le 
ะับ

焉安

$\approx$.

ปั

ปे

य)

จ

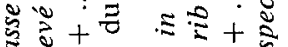

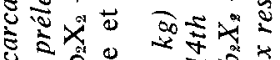

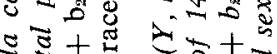

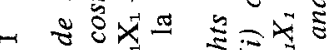

D

बิ

起的焉

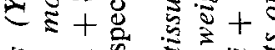
ज和安

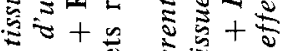

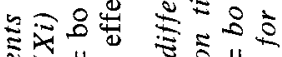
5

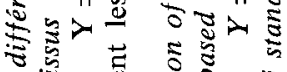
\& केष्ठ

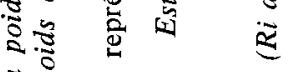
उ

$\approx$ ป

莡 ำ

红

\begin{tabular}{|c|c|c|c|}
\hline 总 & $\underline{=}$ & $\stackrel{m}{a}$ & $\therefore$ \\
\hline 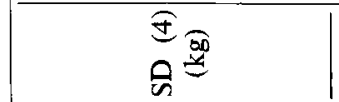 & $\begin{array}{l}\infty \\
\infty \\
\dot{f}\end{array}$ & $\begin{array}{c}3 \\
0 \\
i n\end{array}$ & $\stackrel{\infty}{\sim}$ \\
\hline $\begin{array}{l}\widehat{0} \\
\approx \\
\approx\end{array}$ & $\begin{array}{l}\text { Oे } \\
\text { o. } \\
0\end{array}$ & $\mid \begin{array}{l}\infty \\
\infty \\
0 \\
0 \\
0\end{array}$ & $\begin{array}{l}\text { ठ̊ } \\
\text { ळ. } \\
0\end{array}$ \\
\hline$\sum_{i}^{\infty} \widehat{d}$ & 1 & 1 & $\begin{array}{c}\frac{9}{n} \\
3 \\
3 \\
1\end{array}$ \\
\hline 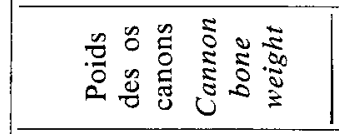 & 1 & 1 & $\begin{array}{l}\hat{n} \\
\text { in } \\
+ \\
+\end{array}$ \\
\hline 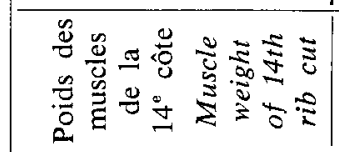 & 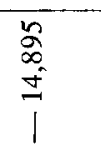 & $\mid \begin{array}{l}0 \\
8 \\
\vdots \\
\hat{n} \\
+\end{array}$ & 1 \\
\hline 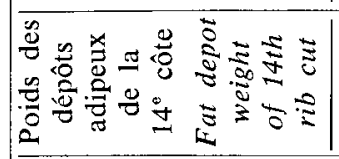 & 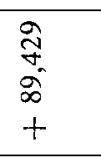 & $\mid$\begin{tabular}{l} 
D \\
\multicolumn{1}{c}{} \\
0 \\
0 \\
1 \\
\end{tabular} & 1 \\
\hline 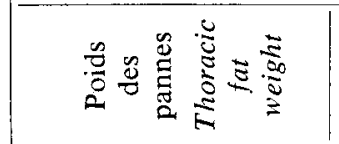 & 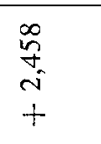 & in & 1 \\
\hline 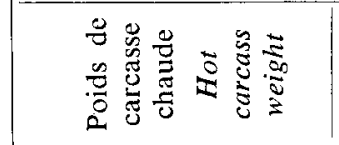 & $\begin{array}{l}\infty \\
8 \\
0 \\
+ \\
+\end{array}$ & $\mid \begin{array}{l}0 \\
\vdots \\
0 \\
+\end{array}$ & $\begin{array}{l}\hat{o} \\
\stackrel{0}{0} \\
+\end{array}$ \\
\hline 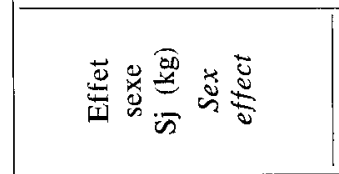 & $\begin{array}{l}\text { sot } \\
\text { o ot } \\
\text { S: } \\
\text { o } \\
\text { in } \\
1+\end{array}$ & $\tilde{z}$ & $\tilde{z}$ \\
\hline
\end{tabular}

$\frac{5}{8}$

$\frac{5}{5}$

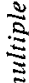

至

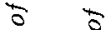

$\stackrel{\square}{\frac{5}{5}}$

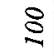

$\approx \quad \Xi$

$\frac{0}{0}$

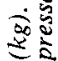

อ

हैं

है:

중

ะ $\approx$

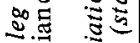

हैं के चี

픈 닝

ปั ป

2.

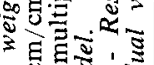

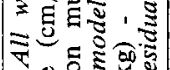

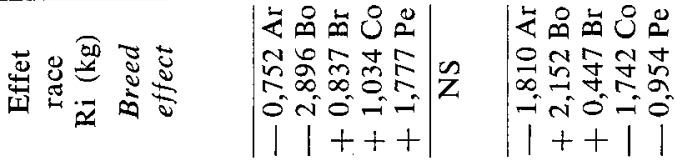

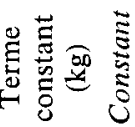

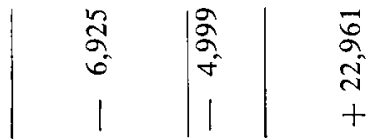

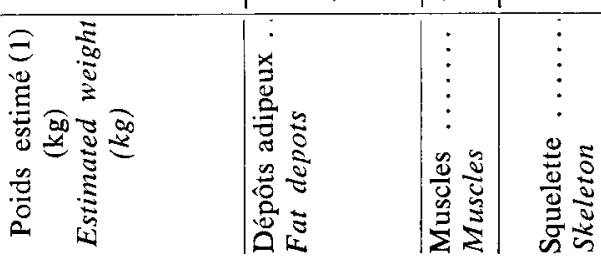




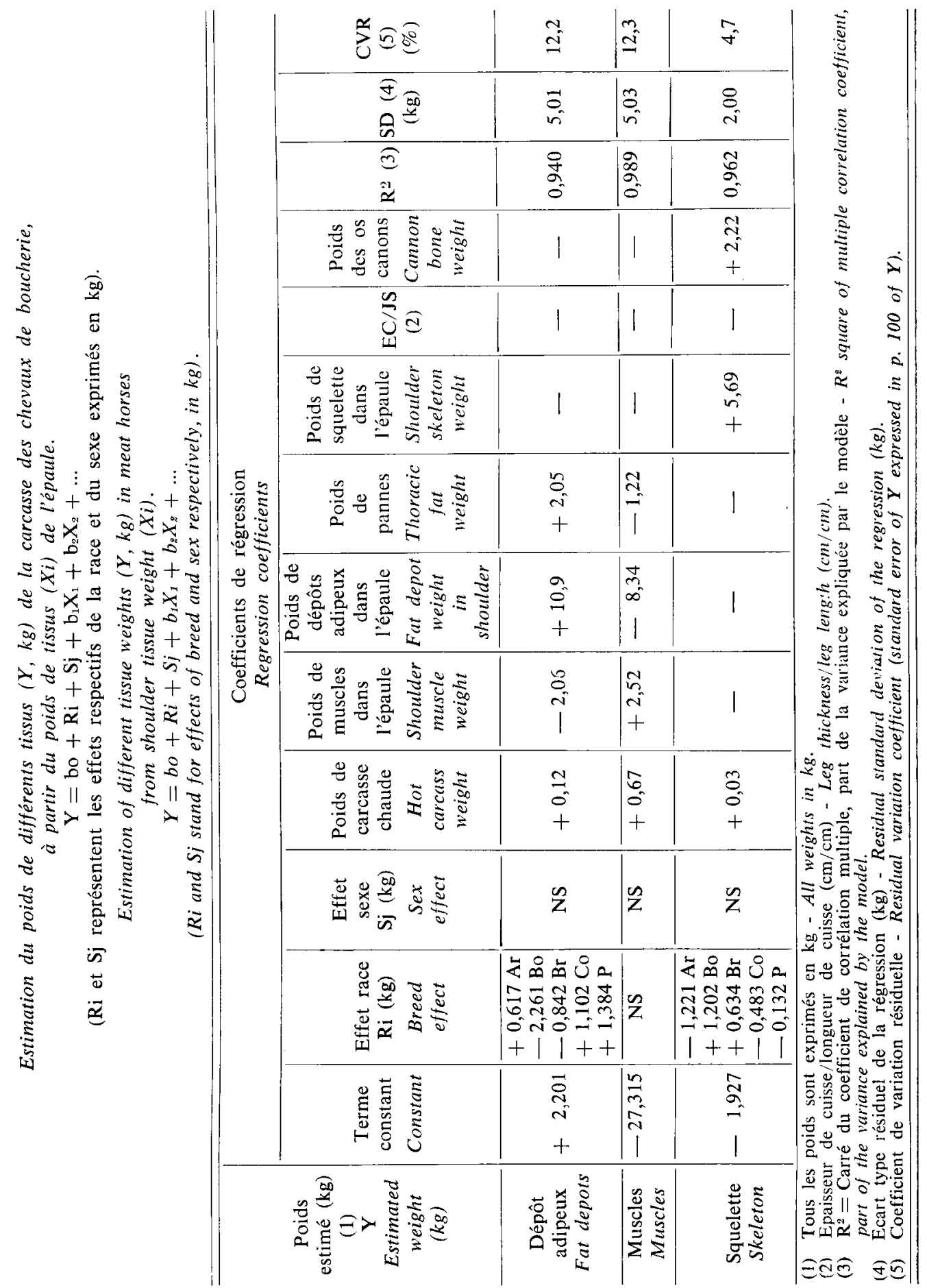


poids des dépôts adipeux et des muscles de l'épaule améliore considérablement cette précision qui devient voisine de $5 \mathrm{~kg}$.

Le sexe a un effet très significatif lorsqu'on estime le poids des dépôts adipeux avec l'équation " $14^{\mathrm{e}}$ côte » $(\mathrm{P}<0,005)$ mais pas d'effet sur l'équation « Epaule». La race a également un effet sur les deux équations d'estimation. Dans les deux cas l'amplitude de ces effets est voisine de $4 \mathrm{~kg}$.

\section{B. Estimation des muscles (tabl. 1 et 2)}

Le poids de la musculature dans la carcasse peut être estimé à $5,0 \mathrm{~kg}$ près soit environ 12 p. 100 en valeur relative ; à partir du poids de carcasse chaude et du poids des pannes ; et respectivement du poids des dépôts adipeux et des muscles de l'épaule $(\mathrm{P}<0,0025)$ ou du poids des dépôts adipeux et des muscles de la $14^{\mathrm{e}}$ côte $(\mathrm{P}<0,005)$.

La race et le sexe n'ont aucun effet significatif sur ces équations. Sur le plan pratique, cela signifie qu'il est possible d'estimer le poids de la musculature de chevaux de format aussi différents que des Comtois et des Percherons en utilisant une seule équation.

\section{Estimation du squelette (tab1. 1 et 2)}

Le poids de carcasse, celui des os canons et la compacité de la cuisse permettent d'estimer le poids du squelette avec un écart-type résiduel égal à $3,3 \mathrm{~kg}$ près soit 7,9 p. 100 en valeur relative. Le poids du squelette de la $14^{\mathrm{e}}$ côte n'apporte pas d'information supplémentaire. En revanche, le poids du squelette de l'épaule améliore très significativement la relation : l'écart-type résiduel $(2 \mathrm{~kg})$ ne représente plus que 4,7 p. $100 \mathrm{du}$ poids du squelette.

Le sexe des animaux n'a pas d'incidence sur les équations, alors que la race a un effet significatif $(P<0,001)$. L'amplitude de la variation entre races de la valeur estimée est égale à $2,5 \mathrm{~kg}$.

\section{Discussion}

Le poids des différents tissus de la carcasse des poulains peut être estimé avec une erreur inférieure à $5 \mathrm{~kg}$ à partir de critères facilement mesurables.

Nos résultats montrent que le poids des muscles est relié plus étroitement au poids des tissus adipeux qu'au poids des muscles de la côte ou de l'épaule. Ce résultat signifie que la composition de la masse maigre est relativement stable. A même état d'engraissement, les poids moyens des muscles et des os ne varient respectivement que de 2 et 6 p. 100 tandis qu'à même poids de carcasse le poids moyen des dépôts adipeux est beaucoup plus variable (12,7 p. 100). Le cheval se comporte à ce titre comme les bovins (ButTerfield, 1965 ; Robelin \& GeAY, 1975). L'estimation de la composition de la carcasse des chevaux revient donc comme chez les bovins à une mesure de leur état d'engraissement. 
L'épaule et la $14^{\text {e }}$ côte peuvent être utilisées indifféremment. La précision des résultats obtenus n'est supérieure avec l'épaule, que dans le cas du squelette. Le choix de la $14^{\circ}$ côte ou de l'épaule dépend donc du coût respectif du prélèvement et de la dissection de ces deux éléments. Dans la majorité des cas, c'est le prélèvement de la $14^{\mathrm{e}}$ côte qui entraîne la moindre dépréciation de la carcasse.

Toutefois, l'utilisation généralisée de telles méthodes a un grand nombre d'animaux pourrait s'avérer trop onéreuse pour des études zootechniques telles que la production de viande chevaline, ou impossible à mettre en œuvre dans le cadre d'études nutritionnelles telles que la mesure de la capacité de mobilisation des réserves corporelles chez la jument allaitante ou le cheval de selle. Dans ce cas il faut disposer de méthodes utilisables sur l'animal vivant, comme l'espace de diffusion de l'eau lourde ou celle du diamètre des adipocytes. Cette dernière méthode qui s'avère intéressante chez les bovins (RoBELIN, 1982) donne également des résultats prometteurs chez les chevaux. On peut également envisager de mesurer l'épaisseur du gras dorsal grâce aux ultra-sons. Westervelt et al. (1976) obtiennent des résultats intéressants chez le cheval. Cependant, une évaluation de cette méthode sur les bovins, mise en place au niveau de la Commission Européenne (BECH ANDERSEN et al., 1982) montre que la précision de cette méthode est encore largement tributaire de l'analyse subjective de l'image faite par l'opérateur.

\author{
Summary \\ Carcass composition of meat horses \\ estimated from the composition of shoulder or 14 th rib cuts
}

Seventy-five colts and fillies from different heavy breeds were slaughtered at 12, 18, 24 and 30 months of age. Carcasses were dissected.

Three criteria likely to be linked to the anatomical carcass composition were measured :

- thoracic fat weight

- cannon-bone weight,

- thigh thickness.

In addition, a 14th rib cut and a shoulder cut were sampled and dissected.

Carcass tissue weight was related to the different criteria measured at slaughter by the progressive regression method. Three equations of different estimations were thus set up for each kind of tissue. Effects of breed and sex were taken into account in the equation to estimate the weight of fat depots. However, only breed was considered for skeleton calculations (tables 1 et 2).

Fat depots and muscle weight were estimated at around $4.9 \mathrm{~kg}$ and $5.0 \mathrm{~kg}$ respectively. The estimation accuracy of skeletal weight was $3.0 \mathrm{~kg}$.

Key words : horse, carcass composition, estimation.

Reçu en juin 1982.

Accepté en novembre 1984. 


\section{Références bibliographiques}

Boccard R., Dumont B.L., Lejebvre J., 1976. Etude de la production de viande chez les ovins. X. Relations entre la composition anatomique des différentes régions corporelles de l'agneau. Ann. Zootech., 25, 95-110.

Bech Andersen B., Busk H., Chadwick J.P., Cuthbertson A., Fursey G.A.J., Jones D.W., LEWIN P., MiLes C.A., OWEN M.G., 1982. CEC supported ultrasonic trial in UK and DK in "In vivo estimation of body composition in beef ». Beretn. Stat. Husdyrbrugs forsog., 524, 13-38.

ButTeRField R.M., 1965. The relationship of carcass measurements and dissection data to beef carcass composition. Res. Vet. Sci., 6, 24-32.

Callow E.H., 1962. The relationship between the weight of a tissue in a single joint and the total weight of the tissue in a side of beef. Anim. Prod., 4, 37-46.

Dumont B.L., Le Guelte P., ArnoulX J., 1961. Etude biométrique des bovins de boucherie. II. Estimation du poids de la musculature chez les bovins charolais. Ann. Zootech., 10, 321-326.

Geay Y., Beranger C., 1969. Estimation de la composition de la carcasse de jeunes bovins à partir de la composition d'un morceau monocostal au niveau de la $11^{\mathrm{e}}$ côte. Ann. Zootech., 18, 65-77.

LEdger H.P., Hutchinson H.G., 1962. The value of the tenth rib as a sample joint of the estimation of lean fat and bone in carcass of East African zebu cattle. J. Agric. Sci., 58, $81-88$.

Martin-Rosset W., Jussiaux M., 1977. Production de poulains de boucherie. Bull. Techn. CRZV Theix, I.N.R.A., 29, 13-21.

Martin-Rosset W., Boccard R., Jussiaux M., Robelin J., Trillaud-Geyl C., 1983. Croissance relative des différents tissus, organes et régions corporelles entre 12 et 30 mois chez le cheval de boucherie de différentes races lourdes. Ann. Zootech., 32, $153-174$.

Prud'hon M., Reyne Y., Garambois X., 1972. Estimation de la composition corporelle d'agneaux Mérinos d'Arles abattus à des stades de croissance compris entre la naissance et un an. Ann. Zootech., 21, 299-309.

Robelin J., GeAY Y., 1975. Estimation de la composition des carcasses de jeunes bovins à partir de la composition d'un morceau monocostal prélevé au niveau de la $11^{\circ}$ côte. I. Composition anatomique de la carcasse. Ann. Zootech., 24, 391-402.

Robelin J., 1982. A note on the estimation in vivo of body fat in cows using deuterium oxide- or adipose- cell size. Anim. Prod., 34, 347-350.

SEEBECK R.M., 1973. The effect of body weight loss on the composition of Braham cross and Africander cross steers. I. Empty body weight, dressed carcass weight, and offal components. J. Agric. Sci., 80, 201-210.

Westervelt R.G., Stouffer J.R., Hintz H.F., Schryver H.F., 1976. Estimating fatness in horses and ponies. J. Anim. Sci., 43, 781-785. 